\title{
Criminologie
}

\section{Le programme de recherches sur la victimisation au Canada}

\section{Gérald J. Léger et John L. Evans}

Volume 13, numéro 1, 1980

Regards sur la victime

URI : https://id.erudit.org/iderudit/017119ar

DOI : https://doi.org/10.7202/017119ar

Aller au sommaire du numéro

Éditeur(s)

Les Presses de l'Université de Montréal

ISSN

0316-0041 (imprimé)

1492-1367 (numérique)

Découvrir la revue

Citer cette note

Léger, G. J. \& Evans, J. L. (1980). Le programme de recherches sur la victimisation au Canada. Criminologie, 13(1), 104-106.

https://doi.org/10.7202/017119ar d'utilisation que vous pouvez consulter en ligne.

https://apropos.erudit.org/fr/usagers/politique-dutilisation/ 
Les premières enquêtes sur la victimisation qui remontent à environ dix ans, ont été menées aux États-Unis dans le but d'obtenir des données sur le « chiffre noir», c'est-à-dire sur les crimes inconnus de la police.

Au Canada on avait attaché relativement peu d'importance aux enquêtes sur la victimisation avant que la Division de la recherche du ministère du Solliciteur général n'entreprenne, il y a environ un an, un programme de recherches méthodiques sur ce sujet déjà étudié cependant, en tant que tel, par la Commission de réforme du droit et par les services administratifs des provinces.

Les enquêtes sur la victimisation qui sont actuellement en cours, doivent permettre d'évaluer le taux de criminalité avec plus de précision que ce n'est le cas, sur la base des statistiques officielles de la police, mais bien qu'on a insisté surtout sur cette approche, ce n'est pas leur but unique. En effet, les recherches qui viennent d'être entreprises au Canada, visent également à fournir des données sur les facteurs associés au risque d'être victime d'un crime, des données utiles et impossibles à obtenir autrement, sur les répercussions du crime, de même que sur la rétroinformation, c'est-à-dire le «feed-back » concernant l'efficacité de divers services de l'appareil de la justice pénale.

C'est ainsi que l'étude de la documentation permet de constater qu'il est possible de mettre au point des enquêtes sur la victimisation susceptibles de fournir de nombreux types de données autres que les taux de criminalité proprement dits.

En premier lieu, et c'est là l'objectif le plus important du point de vue de la prévention du crime, il s'agit de réunir des données sur la portée de diverses caractéristiques personnelles des victimes ainsi que sur les situations ou les comportements qui ont précédé, ou accompagné la commission du délit. Ce sont là, en

* Les enquêtes sur la victimisation au Canada étant relativement nouvelles, nous reproduisons ici à titre exceptionnel, non pas le compte rendu d'un ouvrage, mais un résumé très succinct des travaux de recherches en cours. 
effet, des indicateurs précieux en ce qui a trait au risque ou à la prédisposition des victimes à devenir l'objet d'un acte criminel. La répartition des crimes au sein de la population n'est pas toujours le fruit d'un hasard, il est donc nécessaire de connaître les facteurs liés au risque de victimisation et de mieux planifier, à partir de là, les programmes de prévention destinés à des régions ou à des groupes spécifiques.

On peut y parvenir grâce aux techniques d'échantillonnage utilisées dans les enquêtes sur la victimisation qui permettent une estimation des facteurs de risque, tant pour les victimes que pour les non-victimes. C'est ainsi que la comparaison des victimes et des non-victimes, en ce qui a trait à ces facteurs de risque, peut fournir des renseignements directs sur le lien qui existe entre ces derniers et les possibilités d'être l'objet d'un crime.

A titre d'exemple disons que les statistiques de la police sur les entrées par effraction sont exprimées en fonction de leur nombre par 100000 habitants. Par contre, les enquêtes sur la victimisation comprennent non seulement des données sur le délit concerné, mais aussi sur le nombre de résidences unifamiliales, semi-đétachées, en rangées, jumelées, ou d'appartements dans la population ou juridiction, quartiers ou régions. Par conséquent la relation risque que l'on peut obtenir pour chaque type de logement et lieu de résidence peut indiquer dans quelle mesure il s'agit des facteurs associés à ce type de délit d'entrée par effraction.

D'une manière plus générale les travaux entrepris au Canada vont permettre de réunir des données sur les caractéristiques personnelles des victimes et d'un groupe témoin de non-victimes, ainsi que sur les moyens de protection adoptés par ces deux groupes. A partir de cette masse d'informations, il sera possible de préciser dans quelle mesure les différences relevées ont une importance prédominante, ou au contraire, ne sont pas significatives en tant que telles, compte tenu des modes de comportements, par exemple, de certains types d'individus ou de groupes.

Parallèlement, les données sur la fréquence des crimes signalés et non signalés, sur le risque d'être victime d'un crime, sur les répercussions du crime et sur la performance des divers éléments de l'appareil de la justice pénale, qui découleront des enquêtes sur la victimisation pourront être d'une grande utilité pour de nom- 
breux types d'usagers, tels que les forces policières, les administrateurs de certains services et le public en général.

Ils serviront également, sinon surtout, pour l'élaboration des politiques et des législations préventives concernant au besoin des catégories de délits spécifiques.

Sur le plan empirique, nos deux études méthodologiques ont permis jusqu'à présent de déterminer la possibilité de réaliser les objectifs envisagés et cela à un coût relativement limité grâce à l'utilisation des entrevues par téléphone. Les premières enquêtes effectuées jusqu'à présent sur le terrain semblent confirmer, en outre, que les résultats que nous obtenons correspondent aux buts de la recherche tels que énumérés, très schématiquement il va sans dire, dans cet aperçu global de nos travaux.

Les enquêtes vont donc être poursuivies et on espère pouvoir présenter certaines conclusions au cours de l'année prochaine. 